Editorial

\title{
Notes on crohn's disease and ulcerative colitis in Brazil
}

\section{Editorial}

Crohn'sdiseaseandulcerativecolitis, collectivelyknown as inflammatory bowel diseases (IBD), are an important group of diseases affecting mainly the intestines, characterized by a destructive chronic inflammation which can lead to serious complications, surgery and cancerization. Until a few decades ago, IBDs were regarded as more prevalent in northern hemisphere countries, being rare in those below the Equatorline. However, it is observed in many developing countries an increase in prevalence of IBDs, which have come to occupy an important role among diseases the gastroenterologists and proctologists have to deal with.

In Brazil, although we do not have accurate and reliable statistics on the incidence of IBD, we do have reference centers, located in various regions of the country, all reporting an increasing incidence of the disease. In our own experience, while decades ago only sporadic patients were seen, today an average of one new patient a week come to our outpatient clinic. Taking into account that we are a reference center, it is difficult to define whether this occurs either by a real increase in the incidence of the disease, or a significant improvement in diagnosis, or both factors. Brazilhas a highlymixedpopulation, and we have the disease diagnosed in Caucasians, mestizos, a fro and Japanese descendants. The reseems to be agreement with the wellknown theory of hygiene, (according to which exposure to certain parasites and bacteria during childhood would be protective against the development of IBD), as Brazil, in recent years of the 20th century, has turned to a predominantly urban population, contrary to what occurred at the beginning of that century. It is important for gastroenterologists and emergency medicine professionals to know the disease, especially Crohn's, due to its multifaceted and protracted clinical course, which enables various differential diagnoses. Also pediatricians should be aware of the characteristics of inflammatory bowel disease in children, and abandon the notion that it is a disease of young adults. In our center, we accompanied children under 10 years old, and even a fewmonths old, with Crohn'sdisease.

In our country, private medicine disposes of all technological and therapeutic resources necessary for diagnosis and treatment, but it is expensive and accessible to a minority of the population. The vast majority of Brazilians still depend on the publichealth system, which is deficient and lacking in resources. For example, we found that
Volume 3 Issue 3 - 2015

\author{
Catapani WR, Catapani ANS \\ Faculdade de Medicina do ABC, Brazil
}

Correspondence: Catapani WR, Faculdade de Medicina do ABC,Avenida Principe de Gales 82I 09060-650 Santo Andre/SP Brazil, Email wilson.catapani@fmabc.br

Received: December 13, 2015 | Published: December 17, 2015

many patients who arrive to our center come from distantregions of the country, where even access to a colonoscopy can be difficult.

In short, it is important in Brazil, and presumably in other developing countries, make medical and lay information on IBD more widely known, get reliable statistics on the prevalence and morbidity, making more available the diagnostic methods, and facilitate the distribution of expensive medicines by the government in less assisted regions. The Brazilian Study Group of Inflammatory Bowel Disease (GEDIIB ), the official organ linked to the Brazilian Federation of Gastroenterology, is taking initiatives in this direction.

\section{Acknowledgments}

None.

\section{Conflicts of interest}

Author declares there are no conflicts of interest.

\section{Funding}

None. 